\title{
Vitrification of a monatomic 2D simple liquid
}

\author{
Tomoko Mizuguchi and Takashi Odagaki \\ Department of Physics, Kyushu University, Fukuoka 812-8581 Japan
}

\begin{abstract}
A monatomic simple liquid in two dimensions, where atoms interact isotropically through the Lennard-Jones-Gauss potential [M. Engel and H.-R. Trebin, Phys. Rev. Lett. 98, 225505 (2007)], is vitrified by the use of a rapid cooling technique in a molecular dynamics simulation. Transformation to a crystalline state is investigated at various temperatures and the time-temperature-transformation (TTT) curve is determined. It is found that the transformation time to a crystalline state is the shortest at a temerature $14 \%$ below the melting temperature $T_{m}$ and that at temperatures below $T_{v} \equiv 0.6 T_{m}$ the transformation time is much longer than the available CPU time. This indicates that a long-lived glassy state is realized for $T \leq T_{v}$.
\end{abstract}

\section{Introduction}

Glassy materials have been utilized by human beings since the stone age, but still the transition from a liquid to a glass is far from fully understood on the basis of physics. Difficulty of the understanding lies in the fact that there are two effects in the vitrification process; one is the chemical order due to entangled networks or many constituents, and the other is the topological order due to geometrical jamming.

In fact most of the molecular dynamic (MD) simulations used to observe glass transition have been carried out for liquids with more than two components in order to avoid crystallization [1, 2]. It is known that simple metallic liquids can be vitirified by rapid quenching, but complicated constituents are needed to obtain a long-lived glassy state 3. Colloidal suspensions can be vitrified, but it is known that the system is eventually crystallized 4 . Since most theoretical works [5, 6, 7] assume a simple system, comparison between theory and experiments is rather difficult. Therefore, it is very desirable that we can devise a potential in such a way that atoms interacting with it can be vitrified even if the system consists of one component. Such a potential allows us to separate the contribution of the topological order from the chemical one in the glass formation.

For the purpose of producing a long-lived glassy state of a monatomic simple liquid, we employ the Lennard-Jones-Gauss (LJG) potential [8]. In a previous report [9], we showed that the LJG system can be vitrified in two dimensions and the glassy state at low temperatures is stable for a fairly long time in spite of a simple monatomic potential. In addition, for 3D systems the glass-forming ability of the LJG system has been tested and discussed [10. It is important to note that since any system can be brought into an amorphous state by rapid cooling, one has to investigate if the amorphous state is stable for a long time and shows a glass transition when heated.

In this paper, we investigate the aging-induced crystallization for the monatomic glassforming system with MD simulations. In general simulations, the model system is chosen to favour the clarification of dynamical relaxations under supercooling, while the crystallization is highly suppressed. Therefore, correct discriptions of the relaxation process of the glassforming liquids are still not really understood. We focus attention on this point and proceed with the study of the crystallization process within the model reported here which have a long relaxation time. In Sec.2, we explain our model and methods of MD simulation in detail. Results of the simulation are presented in Sec.3 and we discuss the results in Sec. 4 . 


\section{Model and molecular dynamics simulation}

We consider a monatomic system in two dimensions where atoms interact with each other isotropically via the Lennard-Jones-Gauss (LJG) potential

$$
V(r)=\epsilon_{0}\left\{\left(\frac{r_{0}}{r}\right)^{12}-2\left(\frac{r_{0}}{r}\right)^{6}\right\}-\epsilon \exp \left(-\frac{\left(r-r_{G}\right)^{2}}{2 \sigma^{2}}\right) .
$$

The LJG potential is of a double-well type for most values of the parameters, with the second well of depth $\epsilon$ and width $\sigma$ at position $r_{G}$. We note that the effective potential, similar to the LJG type, exists in metals 11] and has been proposed as a model potential to stabilize certain structures 12 .

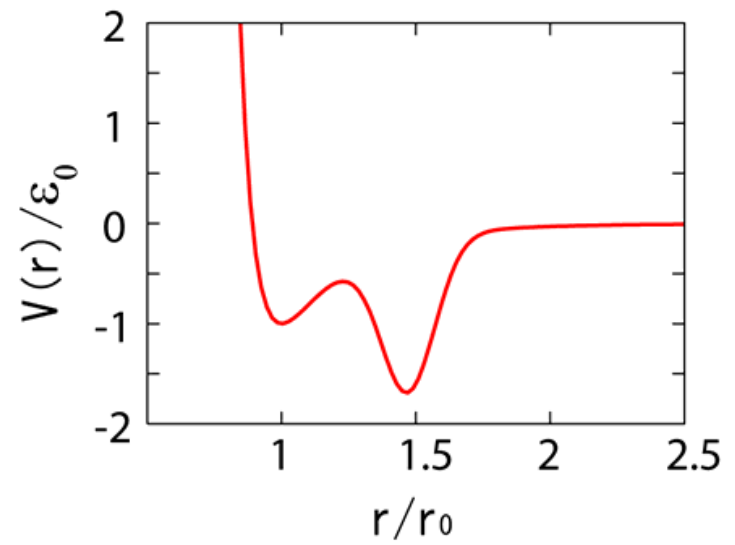

Figure 1: The LJG potential (1) for $r_{G}=1.47 r_{0}, \epsilon=1.5 \epsilon_{0}, \sigma^{2}=0.02 r_{0}^{2}$.

In this paper, we fix the parameters to $r_{G}=1.47 r_{0}, \epsilon=1.5 \epsilon_{0}, \sigma^{2}=0.02 r_{0}^{2}$ and investigate if stable glassy states can be obtained by quenching. Figure 1 shows the LJG potential with these fixed parameters. This set of parameters produces the pentagon-triangle phase in the ground state, where nearest neighbour links form tiling of pentagons, squares and triangles [8].

In the following MD simulation, we set the unit of length, energy and time as $r_{0}, \epsilon_{0}$, $\tau \equiv\left(m r_{0}^{2} / \epsilon_{0}\right)^{1 / 2}$, respectively, and the scaled temperature is denoted by $T^{*} \equiv k_{B} T / \epsilon_{0}$. The number of atoms in the system is $N=1,024$ and the free boundary is used. We employ the Leap-Frog algorithm for the MD simulation with time step $0.01 \tau$.

We first prepared an equilibrium liquid state at $T^{*}=0.4$ above the melting temperature $T_{m}^{*} \simeq 0.34$. Then, we quenched the system to zero temperature instantaneously by removing the kinetic energy. We employ the instantaneous cooling in order to avoid any nucleation during the cooling process which may hinder proper comparison of the crystallization process. After the quenching, we subjected the system to a heat reservoir and allowed it to relax at this temperature. The simulation cell is made large enough so that the system feels zero pressure at all times. Under this free boundary condition, particles on the surface can easily move. Therefore, rearrangement of particles can frequently occur, compared with periodic boundary conditions or a system confined in a small space 13, and cystallization will proceed more quickly and smoothly. We observed the structural transformation in real space as a function of observation time.

\section{Results}

In order to determine the crystallization time, we focus on the formation of three characteristic tiling structures, PPPT, PPP and PPTS, which consist of pentagons, squares and triangles shown in Fig. 2 (a). Figure 2 (b) shows the time dependence of the number of atoms whose surroundings can be identified as the three characteristic tiling structures. The curve (all) shows the number of particles surrounded by any of the pentagons, triangles or squares. This value is related to the potential energy and becomes constant when the system reaches a crystalline state. At relatively high temperatures, the transition from the supercooled liquid to a crystal is rather sharp and we can determine the crystallization time without ambiguity. As temperature is reduced, the transformation tends to be less sharp, 


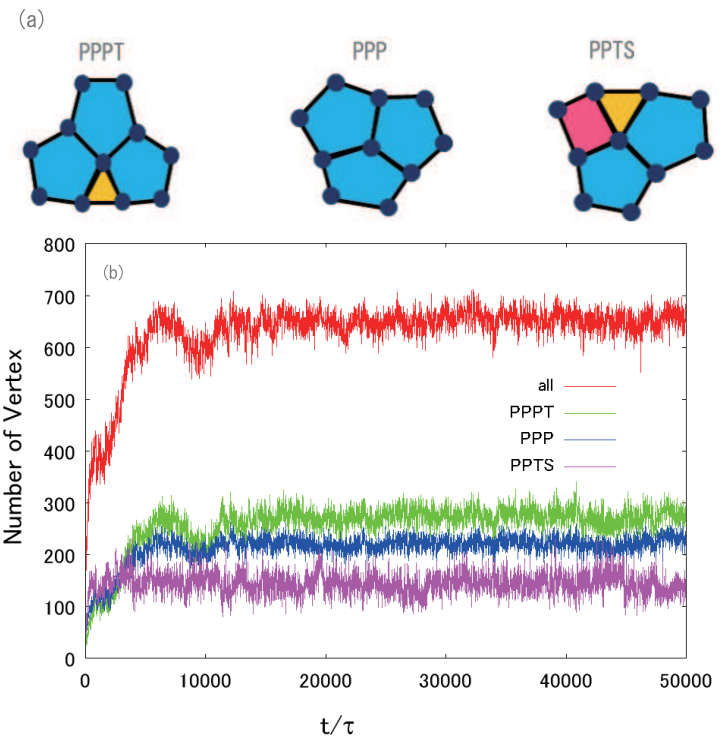

Figure 2: (a) Three charateristic tiling structures. (b) The time dependence of the number of characteristic tiling structures. The curve (all) represents the number of atoms surrounded by any of the pentagon, triangle or square tiles. The curves (PPPT), (PPP) and (PPTS) correspond to the structures shown in (a).

and we determined the transition time with a diffraction pattern as well as the evolution of tilings. Figure 3 shows the crystallization time as a function of temperature which is known as a time-temperature-transformation (TTT) diagram. This diagram indicates that the crystallization time becomes the shortest at $\sim 0.88 T_{m}$ and it is longer than $10^{7} \tau$ at $0.5 T_{m}$. If one uses the value of $m, r_{0}$ and $\epsilon_{0}$ relevant for Ar, this time corresponds to 10 micro seconds.

For comparison, the results for a monatomic Lennard-Jones(LJ) system are also plotted in Fig. 3, where the crystallization time is about $10^{3} \mathrm{MD}$ steps. Consequently, the LJG system has a much longer crystallization time than the LJ system, and we can clearly see the temperature dependence of the TTT diagram in the LJG system. It shows a typical nose shape, which has been observed by experiments for various glass forming materials [3, 14, 15]. A similar TTT diagram is also found by MD simulations with some empirical potentials for metal 16, 17. Our system has a much longer crystallization time than that reported in these papers in spite of the simpler shape of our interatomic potential, if we use the parameters relevant for Ar.

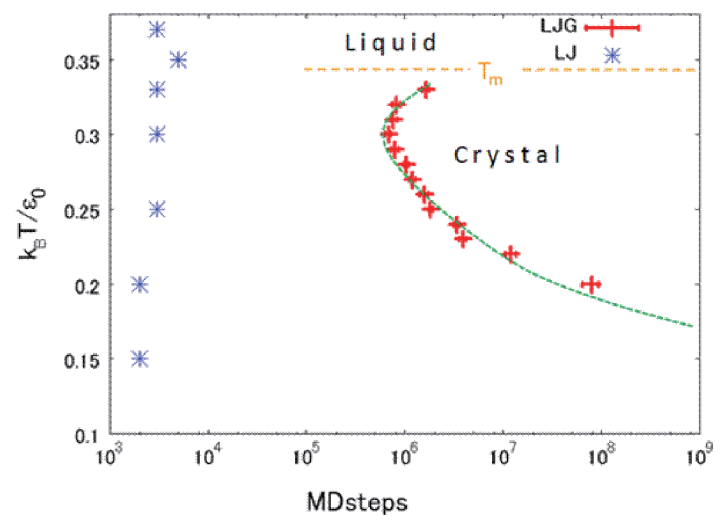

Figure 3: The time-temperature-transformation diagram for LJG and LJ liquids.

In Fig. 4, we show the atomic configurations at two different temperatures, $T^{*}=0.3$ and 0.15 , and two different observation times $t / \tau=3 \times 10^{2}$ and $10^{5}$. We can clearly see that at $T^{*}=0.3$ crystallization has taken place in this time period and that at $T^{*}=0.15$ 
the system keeps the amorphous structure.
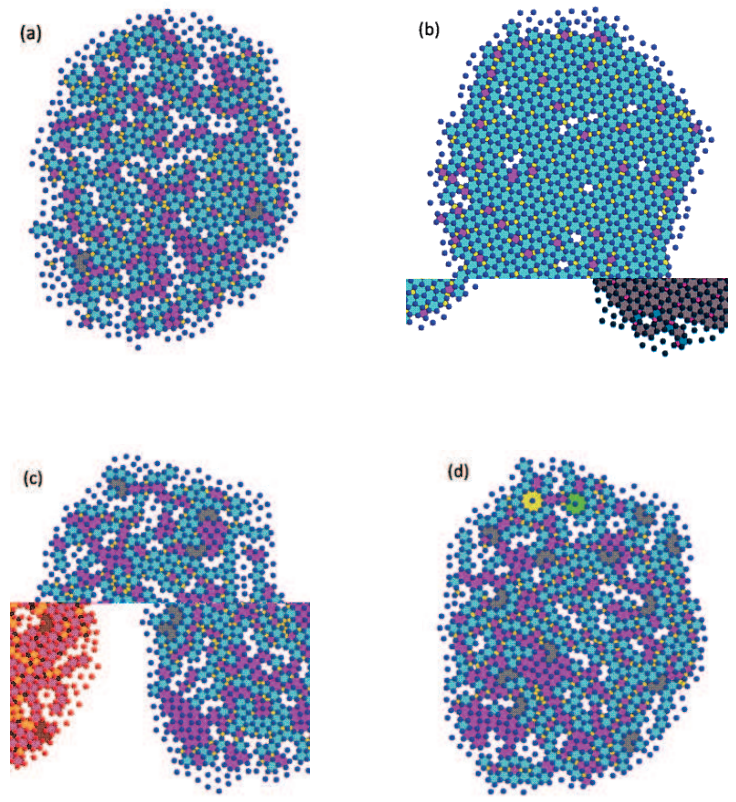

Figure 4: Evolution of the tiling structure. Upper two panels are at $T^{*}=0.3$ and $t / \tau=3 \times 10^{2}(\mathrm{a})$ and $10^{5}(\mathrm{~b})$. Lower two panels are at $T^{*}=0.15$ and $t / \tau=3 \times 10^{2}(\mathrm{c})$ and $10^{5}(\mathrm{~d})$.

\section{Discussion}

We have succeeded in vitrifying a simple monatomic liquid in two dimensions by MD simulation and shown that the glassy state can live a fairly long time at low temperatures when the parameters of the LJG potential are chosen adequately. The Gaussian part of the LJG potential stabilizes the pentagonal configuration and packing of pentagons produces frustration in crystallization. Two competing length scales can have a great impact on the degree of disorder in the system. Also, in 3D systems 10, the glassy state can be formed if the LJG potential has appropriate parameters favoring the formation of an icosahedral local order. The effect of this frustration with packing is the origin of the stability of the glassy state.

The present simulation was carried out at zero pressure. In a previous report 9], we investigated the glass transition of the same system at higher pressure corresponding to a constant denisity $\rho, \rho \pi r_{0}^{2}=1$. We obtained the temperature dependence of the energy while the system was heated at a constant rate, and showed that a transition exists where the specific heat increases suddenly, which can be identified as the glass transition. A full report of the observation will be given in a forth coming paper [18].

The TTT diagram (Fig. 3) indicates that one can keep the glassy state of the LJG system for a fairly long time. Since we employ the free boundary condition in this study, the system can crystallize faster as compared with other confined boundary conditions. In fact, it was found that the amorphous structure of Lennard-Jones Ar confined in a nanoscale pore is more stable than a bulk Ar 13 . Therefore, with other confined boundary conditions, our system will show stronger resistance against crystallization. Due to the stability of glass, this model provides a chance of further study about the glass transition and other related phenomena. In fact, our preliminary investigation 9 showed that the intermediate scattering function of the super cooled liquid state shows two step relaxation similar to those observed by experiments.

It is interesting to note that the LJG system can have crystalline states, quasi-periodic sates and glassy states and the dynamics in these states, as well as change in dynamics during the transformation, can be investigated and that the findings for the LJG system will serve as standards against which theroies can be tested. In particular, relation between the slow dynamics in glassy systems and the phason dynamics in quasi-crystals may be scrutinized. 


\section{Acknowledgement}

We would like to thank Dr. Michael Engel of Stuttgart University for providing us with the software on which most of the simulations and analysis for this work were carried out. This work was supported in part by the Grant-in-Aid for Scientific Research from the Ministry of Education, Culture, Sports, Science and Technology. .

\section{References}

[1] B. Bernu, J. P. Hansen, Y. Hiwatari and G. Pastore, Phys. Rev. A 36, (1987) 4891.

[2] W. Kob and H.-C. Andersen, Phys. Rev. E 52, (1995) 4134.

[3] R. Bush, J. Schroers, and W. H.Wang, MRS Bulletin 32, (2007) 620.

[4] W. van Megen, Phys. Rev. E 76, (2007) 061401.

[5] U. Bengtzelius, W. Götze and A. Sjölander, J. Phys. C 17, (1984) 5915; W. Götze, in "Liquids, Freezing and the Glass Transition" (edited by J. P. Hansen, D. Levesque and J. Zinn-Justin), (North Holland 1989) p. 287; W. Götze, J. Phys.: Condens. Matt. 11, (1999) A1.

[6] T. Odagaki, Phys. Rev. Lett. 75, (1995) 3701; T. Odagaki, T, Yoshidome, A. Koyama, and A. Yoshimori, J. Non-Crys. Solids, 352, (2006) 4843; T. Odagaki and A. Yoshimori, J. Non-Crys. Solids, to appear.

[7] M. Mézard, and G. Parisi, J. Chem. Phys. 111, (1999) 1076.

[8] M. Engel and H.-R. Trebin, Phys. Rev. Lett. 98, (2007) 225505; M. Engel, Ph. D. Thesis (Stuttgart University, 2008).

[9] T. Mizuguchi, T. Odagaki, M. Umezaki, T.Koumyou, and J. Matsui, Conference Proceedings 982 "Complex Systems", (AIP, New York 2008) 234.

[10] Vo Van Hoang and T. Odagaki, Physica B 403, (2008) 3910

[11] Al-Lehyani, M. Widom, Y. Wang, N. Moghadam, G. M. Stocks and J. A. Moriaty, Phys. Rev. B 64, (2001) 075109.

[12] M. Rechtsman, F. Stillinger and S. Torquato, Phys. Rev. Lett. 95, (2005) 228302; Phys. Rev. E 73, (2006) 011406.

[13] K. Nishio, J. Koga, T. Yamaguchi and F. Yonezawa, J. Phys. Soc. Jpn 73, (2004) 627.

[14] H. Senapati, R. K. Kadiyala and C. A. Angell, J. Phys. Chem. 95, (1991) 7050.

[15] S. Mukherjee, J. Schroers, W. L. Johnson and W.-K. Rhim, Phys. Rev. Lett. 94, (2005) 245501.

[16] A. V. Evteev, A. T. Kosilov, E. V. Levchenko and O. B. Logachev J. Exp. Theor. Physics 101, (2005) 521.

[17] Y. Zhang, Li Wang and W. Wang, J. Phys.:Condens. Matter 19, (2007) 196106.

[18] T. Mizuguchi and T. Odagaki, in preparation. 